\title{
Individualized Food-Based Dietary Therapy for Crohn's Disease: Are We Making Progress?
}

\author{
Dale Lee $^{1} \cdot$ David Suskind $^{1}$
}

Published online: 10 February 2016

(c) Springer Science+Business Media New York 2016

Immune dysregulation contributes to the pathophysiology of inflammatory bowel disease (IBD), which can occur in genetically susceptible individuals, likely triggered by environmental exposures, including components of the diet, which in turn has profound effects on the composition of the gut microbiome and on the gastrointestinal epithelium and immune system $[1,2]$. Further, dietary exposures correlate with the incidence of IBD and dietary therapies can effectively induce remission of active inflammation [3]. The primary benefit of dietary therapies in IBD, as either primary or adjunctive therapy, has been the potential to decrease the exposure to immunosuppressive medications and their associated adverse effects.

Although genetic changes do not occur rapidly enough to explain the rising incidence of IBD, changing environmental and dietary exposures are likely contributory. Epidemiologic studies have reported the association between an increased risk of IBD and greater intake of "western" dietary exposures such as total fats, polyunsaturated fatty acids, $\Omega-6$ fatty acids, and meat [4]. Exclusive enteral nutrition (EEN), or a defined-formula diet with exclusion of other foods, effectively induces remission of active Crohn's disease in $\sim 85 \%$ of children [3]. The efficacy of food-based exclusion diets in treating IBD is supported by studies evaluating the specific carbohydrate diet (SCD) and the Crohn's disease exclusion diet (CDED) [5-7] which involve broad dietary exclusions across all study participants without guided individualization.

Dale Lee

dale.lee@seattlechildrens.org

1 Seattle Children's Hospital, Division of Pediatric Gastroenterology, University of Washington, 4800 Sandpoint Way NE, Seattle, WA 98105, USA
The potential mechanisms of action by which the diet impacts IBD may involve the mechanical properties of foods, the delivery of essential nutrients, the composition and function of gut microbiota, alterations in bile acid homeostasis, or reduced exposures to deleterious compounds [8]. Data from a recent study evaluating EEN versus partial enteral nutritional therapy suggest that the efficacy of nutritional therapy is associated with the exclusion of specific dietary components, as opposed to the therapeutic delivery of beneficial nutrients [9]. Although EEN is not effective in adult studies, this may result from poor compliance combined with greater prior exposure to immunosuppressive therapies [8]. Whereas dietary therapy with EEN involves strict food elimination, exclusion diets are more complex to implement and more difficult to monitor for compliance. The restrictive nature of EEN is difficult to maintain while targeted food elimination may be more sustainable. That genetic polymorphisms in the NOD2 gene are associated with differential outcomes in children with Crohn's disease treated with EEN supports the concept that individualization of dietary therapeutic approaches can improve outcomes [10].

In this month's issue of Digestive Disease Sciences, Gunasekera et al. [11] present data from a randomized controlled trial examining the efficacy of what they term an "IgG4-guided elimination diet" in patients with Crohn's disease, in which 16 common foods were evaluated for their effect on IgG4 titers. Individualized food exclusions were divided into two groups: the intervention group with exclusion of four food groups associated with the highest IgG4 titers and the comparison group in which the four food groups associated with the lowest IgG4 titers were excluded. Subjects were followed over 4 weeks; the primary aim was to measure the effect of the dietary intervention on the quality of life (QOL) as measured by the 
Short Inflammatory Bowel Disease Quality of Life Questionnaire (SIBDQ). Secondary aims included evaluation of the change in the clinical disease activity indices as measured by the Crohn's Disease Activity Index (CDAI) and the Harvey-Bradshaw Index (HBI), and evaluation of change in inflammatory biomarkers such as the C-reactive protein (CRP) and fecal calprotectin (FCP). The authors reported that the intervention group had significantly greater declines in SIBDQ, CDAI, and HBI, suggesting that IgG4-guided dietary exclusions have the ability to positively impact QOL and clinical disease activity. In the per-protocol analysis, CRP and FCP, respectively, were statistically similar between the intervention and control groups, agreeing with previous reports that documented a lack of correlation between inflammatory biomarkers, clinical disease activity indices, and QOL measures [12, 13], although the analysis may not have been powered sufficiently to detect a difference among these outcomes.

There are several important considerations when interpreting these study results. Though discrepancies exist between markers of inflammation, clinical disease activity, and QOL, each is an important measure of disease-associated well-being and impact, capturing a distinct facet of the disease. Whereas endoscopic evidence of mucosal healing and steroid-free remission are often thought of as the most meaningful endpoints, QOL and other subjective measures of well-being may capture important attributes of an individual's functioning in the setting of Crohn's disease. Although the results of the study by Gunasekera et al. do not demonstrate a reduction in inflammation, they do support the concept that guided food exclusion may be useful for symptom management. As with other "recommendation-based" dietary trials, the ability to measure dietary adherence is a limitation that has the potential to bias study results, a shortcoming the authors acknowledge. Another limitation is the duration of the elimination diet, since improvement in inflammatory markers may require an intervention lasting more than 4 weeks; EEN studies have commonly evaluated inflammatory parameters at 8-10 weeks. Finally, though IgG4-based guided dietary exclusion may inform guided dietary therapy for IBD, further elucidation of the underlying mechanism by which food exposures are involved in IBD pathogenesis may be required to formulate effective dietary interventions.

Since the study by Gunasekera et al. demonstrates the potential for individualized dietary therapy in IBD, it may inform the methodology for other studies. While existing therapeutic dietary options such as EEN, the SCD, or the CDED can help define individuals as "food responders" or not, Gunasekera's study emphasizes the importance of individualizing dietary therapies. In the future, as the connection among dietary exposures, intestinal inflammation, and the gut microbiome is better understood, dietary therapy is more likely to be individualized. Though certain dietary exposures may have a well-defined, generalized deleterious effect, the effects of some dietary exposures may differ for individuals based upon their genetics, the composition of their gut microbiome, and on underlying immune dysregulation. While EEN involves broad dietary exclusion, some targeted exclusion diets may provide insight into the deleterious effects of specific foods. Although the SCD and CDED have many differences, both share the elimination of foods high in food additives such as emulsifiers, preservatives, and processed meats. This is juxtaposed to EEN, which excludes foods, but includes formulas containing many food additives and other "processed" components. Further epidemiological data may guide more refined hypotheses regarding deleterious food components, and thereafter, clinical trials may be able to demonstrate causal associations.

At present, there is much to learn about how diet affects IBD pathogenesis and therapy. The challenge is formidable but the potential benefit is great. New technology, methodology, and collaborations will be key to forward progress in understanding the link between diet and disease. The interest in using diet as therapy is growing; additional thoughtful studies will likely provide data that will improve outcomes in the treatment of IBD.

\section{Compliance with ethical standards}

Conflict of interest The authors have no financial disclosures pertaining to this manuscript.

\section{References}

1. Lewis JD, Chen EZ, Baldassano RN, et al. Inflammation, antibiotics, and diet as environmental stressors of the gut microbiome in pediatric crohn's disease. Cell Host Microbe. 2015;18:489-500.

2. Kau AL, Ahern PP, Griffin NW, Goodman AL, Gordon JI. Human nutrition, the gut microbiome and the immune system. Nature. 2011;474:327-336.

3. Day AS, Lopez RN. Exclusive enteral nutrition in children with Crohn's disease. World J Gastroenterol. 2015;21:6809-6816.

4. Hou JK, Abraham B, El-Serag H. Dietary intake and risk of developing inflammatory bowel disease: a systematic review of the literature. Am J Gastroenterol. 2011;106:563-573.

5. Suskind DL, Wahbeh G, Gregory N, Vendettuoli H, Christie D. Nutritional therapy in pediatric Crohn disease: the specific carbohydrate diet. J Pediatr Gastroenterol Nutr. 2014;58:87-91.

6. Cohen SA, Gold BD, Oliva S, et al. Clinical and mucosal improvement with specific carbohydrate diet in pediatric Crohn disease. J Pediatr Gastroenterol Nutr. 2014;59:516-521.

7. Sigall-Boneh R, Pfeffer-Gik T, Segal I, Zangen T, Boaz M, Levine A. Partial enteral nutrition with a Crohn's disease exclusion diet is effective for induction of remission in children and young adults with Crohn's disease. Inflamm Bowel Dis. 2014;20:1353-1360.

8. Lee D, Albenberg L, Compher C, et al. Diet in the Pathogenesis and Treatment of Inflammatory Bowel Diseases. Gastroenterology. 2015. 
9. Lee D, Baldassano RN, Otley AR, et al. Comparative effectiveness of nutritional and biological therapy in North American children with active Crohn's disease. Inflamm Bowel Dis. 2015.

10. Frivolt K, Schwerd T, Werkstetter KJ, et al. Repeated exclusive enteral nutrition in the treatment of paediatric Crohn's disease: predictors of efficacy and outcome. Aliment Pharmacol Ther. 2014;39:1398-1407.

11. Gunasekeera, V, Mendall, MA, Chan, D, Kumar, D. Treatment of Crohn's disease with an IgG4-guided exclusion diet: a randomized controlled trial. Dig Dis Sci. (Epub ahead of print). doi:10. 1007/s10620-015-3987-z.
12. Peyrin-Biroulet L, Reinisch W, Colombel JF, et al. Clinical disease activity, C-reactive protein normalisation and mucosal healing in Crohn's disease in the SONIC trial. Gut. 2014;63:88-95.

13. Afzal NA, Van Der Zaag-Loonen HJ, Arnaud-Battandier F, et al. Improvement in quality of life of children with acute Crohn's disease does not parallel mucosal healing after treatment with exclusive enteral nutrition. Aliment Pharmacol Ther. 2004;20:167-172. 